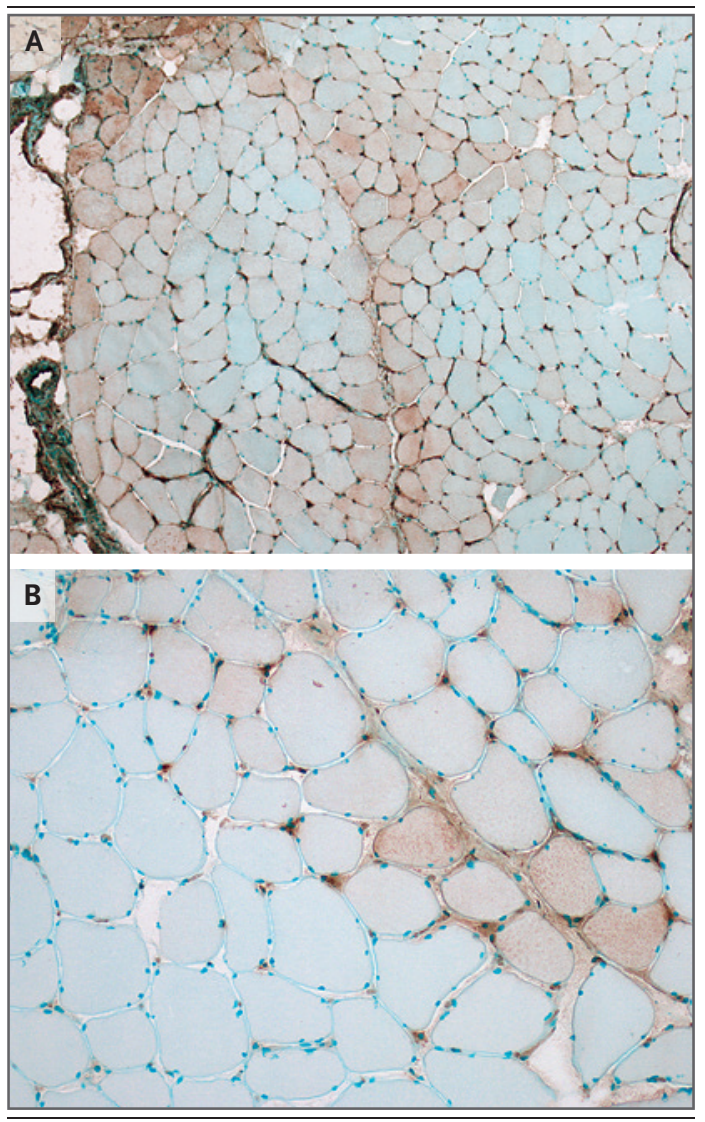

interferon in tissue up-regulates other proteins that are toxic to endothelial cells, muscle, and the lung. The findings in the muscle-biopsy specimen from this patient suggest that his SARSCoV-2 myopathy may also have been due to type I interferonopathy.
Figure 1. Immunohistochemical Analysis of a Biopsy Specimen from the Left Deltoid Muscle in a Patient with Covid-19.

Panel A shows abnormal expression of major-histocompatibility-complex (MHC) class I antigen on the sarcolemma and sarcoplasm of muscle fibers, with a predilection for perifascicular muscle fibers. Expression of MHC class I antigen on capillaries is normal. Panel $B$ shows abnormal expression of myxovirus resistance protein $\mathrm{A}(\mathrm{MxA})$ on sarcolemma and sarcoplasm of muscle fibers, with a predilection for perifascicular muscle fibers, and on capillaries. MxA is not normally expressed on capillaries. Abnormal expression of MxA is seen with type I interferonopathies.

Giovanna S. Manzano, M.D. Jared K. Woods, M.D., Ph.D. Anthony A. Amato, M.D.

Brigham and Women's Hospital Boston, MA gmanzano@partners.org

Disclosure forms provided by the authors are available with the full text of this letter at NEJM.org.

This letter was published on November 20, 2020, at NEJM.org.

1. Guidon AC, Amato AA. COVID-19 and neuromuscular disorders. Neurology 2020;94:959-69.

2. Guan W-J, Ni Z-Y, Hu Y, et al. Clinical characteristics of coronavirus disease 2019 in China. N Engl J Med 2020;382:1708-20. 3. Li L-Q, Huang T, Wang Y-Q, et al. COVID-19 patients' clinical characteristics, discharge rate, and fatality rate of metaanalysis. J Med Virol 2020;92:577-83.

4. Zhang H, Charmchi Z, Seidman RJ, Anziska Y, Velayudhan V, Perk J. COVID-19-associated myositis with severe proximal and bulbar weakness. Muscle Nerve 2020;62:E57-E60.

5. Pulia MS, O'Brien TP, Hou PC, Schuman A, Sambursky R. Multi-tiered screening and diagnosis strategy for COVID-19: a model for sustainable testing capacity in response to pandemic. Ann Med 2020;52:207-14.

DOI: $10.1056 /$ NEJMc2031085

\title{
Effect of Diet versus Gastric Bypass on Metabolic Function in Diabetes
}

TO THE EDITOR: In their article, Yoshino et al. (Aug. 20 issue) $)^{1}$ conclude that weight loss is the main mechanism by which gastric bypass improves glucose homeostasis. Given the limitations and findings of this study, this conclusion seems to be overreaching. First, type 2 diabetes is heterogeneous; small, nonrandomized studies involving participants who are not matched for disease severity at baseline are prone to bias. In fact, in Yoshino et al.'s study, participants who underwent gastric bypass (surgery group) used more medications and had higher fasting insulin and lower adiponectin levels than did participants in the diet group - a finding that suggests differences in disease severity, pathophysiology, or both. In addition, octreotide blocks gut-hormone release and other gastrointestinal mechanisms ${ }^{2}$; its use during clamp studies may have negated weight-independent effects of gastric surgery. Furthermore, the use of a very-low-calorie diet rapidly reverses hyperglycemia ${ }^{3}$ but takes longer than gastric bypass to lead to an $18 \%$ reduction in 
body weight. Thus, longer exposure to reduced glucotoxicity in the diet group than in the surgery group may have influenced beta-cell function and insulin sensitivity, ${ }^{4}$ which were key outcome measures.

Finally, gastric bypass was associated with clinically significant and distinct changes in critical mechanisms of glucose metabolism, including gut hormones, bile acids, branched-chain amino acids, microbiota, and peak response of insulin to meal ingestion. These effects are arguably weight-independent, ${ }^{5}$ and it is unjustified to dismiss them as irrelevant.

Francesco Rubino, M.D.

King's College London

London, United Kingdom

Ricardo V. Cohen, M.D.

Oswaldo Cruz German Hospital

São Paulo, Brazil

ricardo.cohen@haoc.com.br

Dr. Rubino reports receiving consulting fees and grants from Ethicon and Medtronic, receiving consulting fees from Novo Nordisk, and serving on scientific advisory boards for GI Dynamics and Keyron; and Dr. Cohen, receiving speaker's fees and grants from Medtronic and Johnson \& Johnson, receiving speaker's fees from Jansen Pharmaceuticals, and serving on scientific advisory boards for GI Dynamics and Keyron. No other potential conflict of interest relevant to this letter was reported.

1. Yoshino M, Kayser BD, Yoshino J, et al. Effects of diet versus gastric bypass on metabolic function in diabetes. $\mathrm{N}$ Engl J Med 2020;383:721-32.

2. Harris AG. Somatostatin and somatostatin analogues: pharmacokinetics and pharmacodynamic effects. Gut 1994;35:Suppl: S1-S4.

3. Perry RJ, Peng L, Cline GW, et al. Mechanisms by which a very-low-calorie diet reverses hyperglycemia in a rat model of type 2 diabetes. Cell Metab 2018;27(1):210-217.e3.

4. Sutton EF, Beyl R, Early KS, Cefalu WT, Ravussin E, Peterson CM. Early time-restricted feeding improves insulin sensitivity, blood pressure, and oxidative stress even without weight loss in men with prediabetes. Cell Metab 2018;27(6):1212-1221.e3.

5. Pop LM, Mari A, Zhao TJ, et al. Roux-en-Y gastric bypass compared with equivalent diet restriction: mechanistic insights into diabetes remission. Diabetes Obes Metab 2018;20:1710-21.

DOI: 10.1056/NEJMc2030030

TO THE EDITOR: The study by Yoshino et al. has certain limitations and confounders that may result in an unsubstantiated dismissal of important weight-independent mechanisms of glucose control after procedures that bypass the proximal gut. The study was underpowered and had unequal proportions of participants who withdrew. The differential caloric intake was an important confounder - a very-low-calorie diet for
23 weeks in the diet group, as compared with the surgery group, in which participants reached the weight-loss goal at 13 weeks (after which a more liberal diet was offered for 3 weeks in order to prevent further weight loss). The authors overlook the profound changes in glucose homeostasis preceding marked weight loss after gastric bypass. It is plausible that gut-mediated, weight loss-independent effects were masked by this differential caloric restriction, which results in multiple intestinal adaptations promoting insulinsensitivity improvement by correcting gut dysbiosis and enterocyte tight-junction function, with decreasing liver and visceral fat inflammation, proliferation of small-intestinal stem cells, and favorable alterations in the enteric nervous system functions that affect the gut-central nervous system metabolic axis..$^{1-4}$ The results of the study by Yoshino et al. do not rule out intestinally mediated factors that induce insulin resistance, which include potentially formidable therapeutic targets for metabolic disease. ${ }^{5}$

Barham K. Abu Dayyeh, M.D., M.P.H.

Mayo Clinic

Rochester, MN

abudayyeh.barham@mayo.edu

Dr. Abu Dayyeh reports receiving consulting fees from Metamodix, BFKW, and DyaMx, consulting fees and grant support from Boston Scientific, lecture fees and grant support from Medtronic, grant support from Apollo Endosurgery and USGI Medical, and lecture fees from Johnson \& Johnson and holding a patent (WO2017062753A1) and a pending patent (US15/766,604), both entitled "Electroporation for Obesity or Diabetes Treatment." No other potential conflict of interest relevant to this letter was reported.

1. Duca FA, Bauer PV, Hamr SC, Lam TK. Glucoregulatory relevance of small intestinal nutrient sensing in physiology, bariatric surgery, and pharmacology. Cell Metab 2015;22:367-80.

2. Harris TE, Thorner MO. Caloric restriction in mTORC1 control of intestinal homeostasis. Cell Metab 2012;16:6-8.

3. van Baar ACG, Nieuwdorp M, Holleman F, Soeters MR, Groen AK, Bergman JJGHM. The duodenum harbors a broad untapped therapeutic potential. Gastroenterology 2018;154:773-7.

4. West JA, Tsakmaki A, Huang JHS, et al. Proximal and distal gut mucosa adapt differently to Westernized diet, promoting an insulin-resistant dysmetabolic state. October 29, 2019 (https:/ www.biorxiv.org/content/10.1101/822122v1.full). preprint.

5. van Baar ACG, Holleman F, Crenier L, et al. Endoscopic duodenal mucosal resurfacing for the treatment of type 2 diabetes mellitus: one year results from the first international, open-label, prospective, multicentre study. Gut 2020;69:295-303.

DOI: 10.1056/NEJMc2030030

TO THE EDITOR: The study by Yoshino et al. provides important data. However, the authors over- 


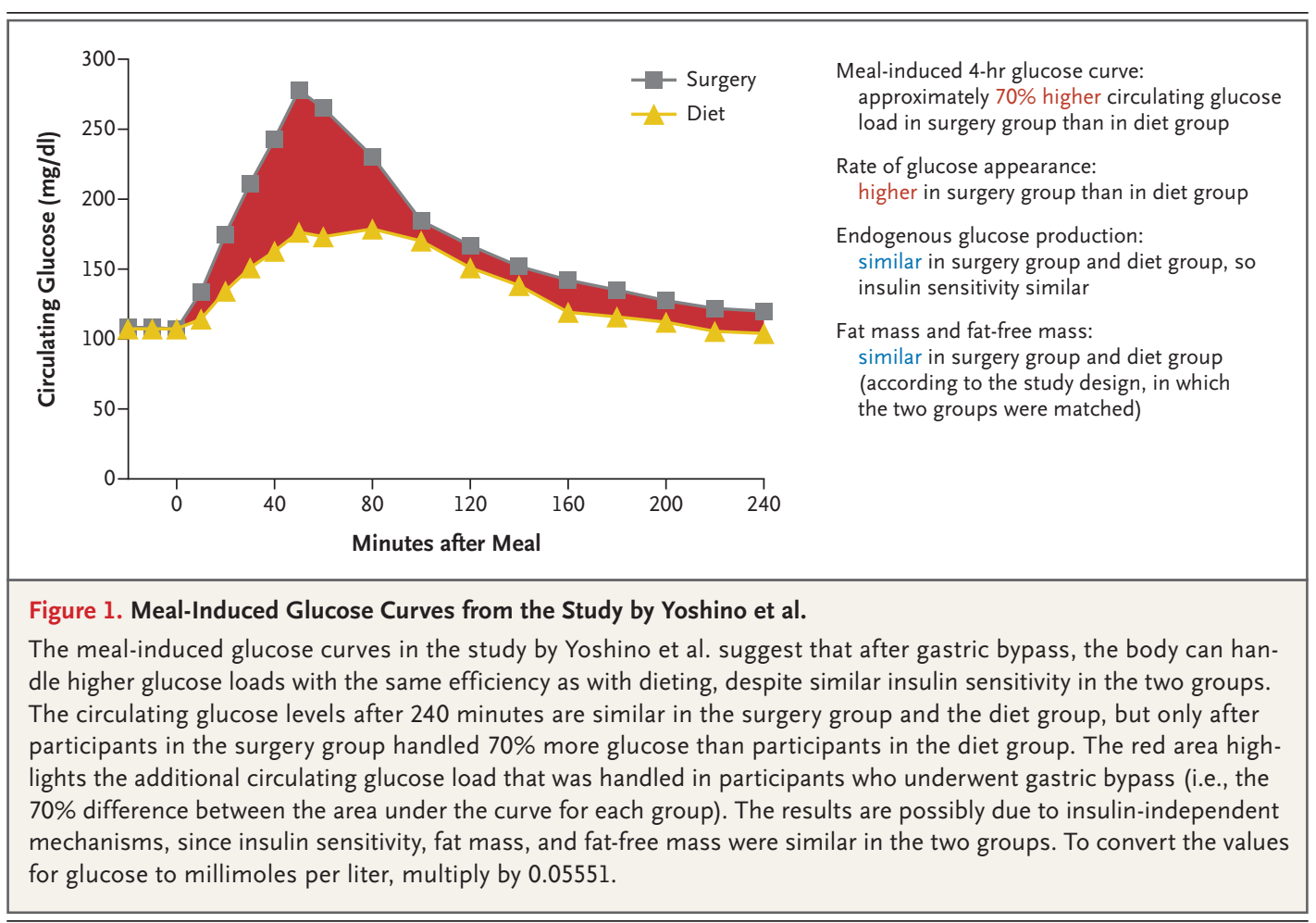

look the very different patterns of the curves for glucose and free fatty acids in the diet group and the surgery group. The data show that weight loss could account for an improvement in insulin sensitivity, but the data also suggest a surgeryspecific, insulin-independent mechanism; the superimposed curves from Figure $1 \mathrm{~A}$ of the article by Yoshino et al. are shown here in Figure 1. In addition, the glucose and insulin curves in the surgery group resemble the patterns observed in high-glycemic-index diets. Unlike these diets, however, gastric bypass seems to protect against diabetes totally. This finding cannot be attributed to weight loss, nor can the distinct patterns regarding free fatty acids (Fig. 2B of the article by Yoshino et al.), which suggest enhanced mealinduced suppression followed by faster lipolysis in the surgery group than in the diet group.

Gastric bypass and dieting may modulate different pathways that result in the same final homeostasis without further improvement. Consider the analogy of two drugs that induce similar improvements in clinically relevant end points (e.g., diabetes) by means of very different mechanisms. That could be the case for surgery as compared with dieting and may also explain the sustainability of the antidiabetic effects of gastric bypass. Look-alike does not necessarily mean work-alike.

Nima Saeidi, Ph.D.

Massachusetts General Hospital

Boston, MA

Margaret Stefater, M.D., Ph.D.

Nicholas Stylopoulos, M.D.

Boston Children's Hospital

Boston, MA

nicholas.stylopoulos@childrens.harvard.edu

No potential conflict of interest relevant to this letter was reported.

DOI: 10.1056/NEJMc2030030

TO THE EDITOR: Yoshino et al. found that an $18 \%$ reduction in body weight improves insulin sensitivity similarly after gastric bypass or dieting in participants with obesity and type 2 diabetes. They conclude that their study results "challenge the current belief that upper gastrointestinal bypass has clinically meaningful effects on key metabolic factors involved in glucose homeostasis and the pathogenesis of diabetes that are independent of weight loss."

Nevertheless, in a recent article ${ }^{1}$ the same 
research group reported that, after a $20 \%$ reduction in body weight, biliopancreatic diversion improved insulin sensitivity significantly more than gastric bypass. How do the authors reconcile these differing results? Furthermore, we note that the participants in the diet group in the study conducted by Yoshino et al. had less severe diabetes than those in the surgery group and appeared to use fewer medications, or no medications (mean $[ \pm S D]$ diabetes medication score [on a scale from 0 to 3.57, with a higher score indicating a greater number of medications, a higher dosage of medications, or both], $0.93 \pm 0.55$ vs. $1.64 \pm 1.15)$.

Lidia Castagneto-Gissey, M.D.
Giovanni Casella, M.D.

Sapienza University of Rome

Rome, Italy

Geltrude Mingrone, M.D., Ph.D.

King's College London

London, United Kingdom

geltrude.mingrone@kcl.ac.uk

No potential conflict of interest relevant to this letter was reported.

1. Harris L-A, Kayser BD, Cefalo C, et al. Biliopancreatic diversion induces greater metabolic improvement than Roux-en-Y gastric bypass. Cell Metab 2019;30(5):855-864.e3.

DOI: 10.1056/NEJMc2030030

THE AUthors RePLY: Rubino and Cohen raise important concerns. Our study was small, yet it was powered to detect clinically important differences in hepatic insulin sensitivity (primary outcome). All the secondary outcomes (metabolic response to mixed-meal ingestion; integrated 24-hour plasma glucose, free fatty acid, and insulin profiles; whole-body insulin sensitivity; and beta-cell function) favored the diet group over the surgery group - an unlikely finding if gastric bypass were superior to dieting. We performed a post hoc (exploratory) noninferiority analysis and found that the upper boundary of the $95 \%$ confidence interval for the maximum possible greater benefit of surgery was not clinically significant for all the secondary outcomes (e.g., insulin-stimulated glucose disposal would at most be $1.10 \mu \mathrm{mol}$ per kilogram of fat-free body mass per minute greater after surgery than after dieting). Moreover, our findings are consistent with those of previous studies that used other methods to compare the metabolic benefits of surgery with those in a weight loss-matched group. ${ }^{1,2}$ Octreotide is unlikely to influence assessment of insulin sensitivity after surgery because intestinal hormones are not stimulated during a hyperinsulinemic-euglycemic clamp procedure, which involves glucose infusion, not ingestion. The additional 7 weeks that were needed for weight loss in the diet group were unlikely to have enhanced weight-loss benefits; weight was constant for 3 weeks before testing, and the amount, not rate, of weight loss determines metabolic improvements. ${ }^{3}$

We do not agree that diabetes was more severe in the surgery group than in the diet group. The diabetes medication score that was used in our trial does not measure severity reliably because medications were determined by each participant's physician; indeed, the glycated hemoglobin level was lower in the surgery group than in the diet group (7.2\% vs. $8.0 \%)$. The between-group differences in the insulin and adiponectin levels were not statistically or clinically meaningful, and the duration of diabetes and other metabolic variables, including the baseline values for all main study outcomes, were nearly identical in the two groups. Although changes in bile acids, branched-chain amino acids, and the microbiome differed between the two groups, no clinically important metabolic effects were involved.

Abu Dayyeh proposes unique mechanisms for glucose control after surgery. However, it is important to determine that weight loss-independent benefits exist before one searches for mechanisms. Several studies have shown that improvement in glucose homeostasis before the occurrence of significant weight loss is due to the marked decrease in caloric intake after surgery.,5

We agree with Saeidi and colleagues that the postprandial plasma profile after surgery resembles that of a high-glycemic-index meal, but we do not understand why this means that surgeryinduced benefits are not due to weight loss. If surgery causes benefits by means of other mechanisms, then it must also attenuate the benefits of weight loss itself because the metabolic outcomes were similar. There was no between-group difference in the amount of glucose "handled" after mixed-meal ingestion, which is determined by the amount of ingested glucose 
delivered into the circulation, not by plasma glucose concentrations.

We agree with Castagneto-Gissey and colleagues that biliopancreatic diversion has greater metabolic benefits than gastric bypass surgery. However, that does not mean that gastric bypass surgery is better than diet alone.

Mihoko Yoshino, M.D., Ph.D.

Brandon D. Kayser, Ph.D.

Samuel Klein, M.D.

Washington University School of Medicine

St. Louis, MO

sklein@wustl.edu

Since publication of their article, the authors report no further potential conflict of interest.

1. Jackness C, Karmally W, Febres G, et al. Very low-calorie diet mimics the early beneficial effect of Roux-en-Y gastric bypass on insulin sensitivity and $\beta$-cell function in type 2 diabetic patients. Diabetes 2013;62:3027-32.

2. Holter MM, Dutia R, Stano SM, et al. Glucose metabolism after gastric banding and gastric bypass in individuals with type 2 diabetes: weight loss effect. Diabetes Care 2017;40:7-15.

3. Kuk JL, Christensen RAG, Wharton S. Absolute weight loss, and not weight loss rate, is associated with better improvements in metabolic health. J Obes 2019;2019:3609642.

4. Isbell JM, Tamboli RA, Hansen EN, et al. The importance of caloric restriction in the early improvements in insulin sensitivity after Roux-en-Y gastric bypass surgery. Diabetes Care 2010; 33:1438-42.

5. Pories WJ, Swanson MS, MacDonald KG, et al. Who would have thought it? An operation proves to be the most effective therapy for adult-onset diabetes mellitus. Ann Surg 1995;222: $339-50$.

DOI: 10.1056/NEJMc2030030

Correspondence Copyright @ 2020 Massachusetts Medical Society.

\section{INSTRUCTIONS FOR LETTERS TO THE EDITOR}

Letters to the Editor are considered for publication, subject to editing and abridgment, provided they do not contain material that has been submitted or published elsewhere.

Letters accepted for publication will appear in print, on our website at NEJM.org, or both.

Please note the following:

- Letters in reference to a Journal article must not exceed 175 words (excluding references) and must be received within 3 weeks after publication of the article.

- Letters not related to a Journal article must not exceed 400 words.

- A letter can have no more than five references and one figure or table.

- A letter can be signed by no more than three authors.

- Financial associations or other possible conflicts of interest must be disclosed. Disclosures will be published with the letters. (For authors of Journal articles who are responding to letters, we will only publish new relevant relationships that have developed since publication of the article.)

- Include your full mailing address, telephone number, fax number, and email address with your letter.

- All letters must be submitted through our online submission system at NEJM.org.

Letters that do not adhere to these instructions will not be considered. We will notify you when we have made a decision about possible publication. Letters regarding a recent Journal article may be shared with the authors of that article. We are unable to provide prepublication proofs. Submission of a letter constitutes permission for the Massachusetts Medical Society, its licensees, and its assignees to use it in the Journal's various print and electronic publications and in collections, revisions, and any other form or medium. authors.NEJM.org

To submit a meeting notice: meetingnotices@NEJM.org The Journal's web pages: NEJM.org 